\title{
Real-Time Reconstruction of Arbitrary Slices for Quantitative and In Situ 3D Characterization of Nanoparticles
}

\author{
Hans Vanrompay, Jan-Willem Buurlage, Daniël M. Pelt, Vished Kumar, Xiaolu Zhuo, \\ Luis M. Liz-Marzán, Sara Bals, * and K. Joost Batenburg*
}

\begin{abstract}
A detailed 3D investigation of nanoparticles at a local scale is of great importance to connect their structure and composition to their properties. Electron tomography has therefore become an important tool for the 3D characterization of nanomaterials. 3D investigations typically comprise multiple steps, including acquisition, reconstruction, and analysis/quantification. Usually, the latter two steps are performed offline, at a dedicated workstation. This sequential workflow prevents on-the-fly control of experimental parameters to improve the quality of the $3 \mathrm{D}$ reconstruction, to select a relevant nanoparticle for further characterization, or to steer an in situ tomography experiment. Here, an efficient approach to overcome these limitations is presented, based on the real-time reconstruction of arbitrary 2D reconstructed slices through a 3D object. Implementation of this method may lead to generalized implementation of electron tomography for routine nanoparticle characterization in 3D.
\end{abstract}

\section{Introduction}

Nanoparticles are important for a wide range of applications because of their unique properties, which are in general

H. Vanrompay, Prof. S. Bals

Electron Microscopy for Materials Science (EMAT)

University of Antwerp

Groenenborgerlaan 171, Antwerp 2020, Belgium

E-mail: sara.bals@uantwerpen.be

H. Vanrompay, Prof. S. Bals

NANOlab Center of Excellence

University of Antwerp

Antwerp 2020, Belgium

J.-W. Buurlage, Dr. D. M. Pelt, Prof. K. J. Batenburg

Centrum Wiskunde \& Informatica (CWI)

Science Park 123, Amsterdam XG 1098, The Netherlands

E-mail: k.j.batenburg@cwi.nl

V. Kumar, Dr. X. Zhuo, Prof. L. M. Liz-Marzán

$\mathrm{CIC}$ biomaGUNE and Ciber-BBN

Basque Research and Technology Alliance (BRTA)

Paseo de Miramón 182, Donostia-San Sebastian 20014, Spain

Prof. L. M. Liz-Marzán

Ikerbasque

Basque Foundation for Science

Bilbao 48011, Spain

Prof. K. J. Batenburg

Leiden Institute of Advanced Computer Science (LIACS)

Leiden University

Niels Bohrweg 1, Leiden, CA 2333, The Netherlands

The ORCID identification number(s) for the author(s) of this article can be found under https://doi.org/10.1002/ppsc.202000073.

DOI: 10.1002/ppsc.202000073 strongly connected to their 3D structure $^{[1-10]}$ Heterostructures, in which several compounds are combined within a single nano-object, provide even more flexibility to tune their final properties. For example, bimetallic nanoparticles can display superior properties compared to their monometallic counterparts. ${ }^{[1-13]}$ To understand the connection between structure/ composition and properties, nanoparticles are often investigated by transmission electron microscopy (TEM). Although TEM has become an indispensable tool for studying nanomaterials, it remains difficult to perform a 3D characterization. Indeed, conventional TEM provides 2D projection images of 3D objects, therefore missing a wealth of information. Electron tomography was developed to overcome this issue. ${ }^{[14-17]}$

In 2003, Midgley et al. combined high angle annular dark field scanning TEM (HAADF-STEM) with tomography, ${ }^{[18,19]}$ which has since been successfully applied to investigate a broad variety of nanostructures. ${ }^{[20-24]}$

During a typical electron tomography experiment, a series of 2D projection images are collected along various tilt angles, to cover an angular range that is as large as possible. After alignment of the tilt series, they serve as the input to a mathematical algorithm that reconstructs the 3D structure of the object. Although the acquisition of a tilt series can be automated, it can take (many) hours to obtain all images, depending on the complexity of the experiment. In addition, both the alignment and the reconstruction of the acquired projection images are carried out through offline post-processing procedures, performed at a dedicated workstation. These steps are computationally demanding, leading to a total data processing time of at least $1 \mathrm{~h}$. To dramatically accelerate the acquisition of tilt series, socalled "fast tomography" was recently introduced in both TEM and HAADF-STEM modes..$^{[25-27]}$ The methodology is based on continuously tilting the holder and simultaneously acquiring projection images, ideally while focusing and tracking the particle at the same time.

Fast HAADF-STEM tomography enables a new range of experiments, during which the dynamic behavior of nanoparticles can be probed in 3D. For example, recently this technique was combined with in situ heating to investigate the thermal stability of $\mathrm{Au}$ and $\mathrm{Au} / \mathrm{Pd}$ nanoparticles. ${ }^{[27,28]}$ These experiments are at the state of the art with respect to acquisition time, and we were able to record a full HAADF-STEM tilt series within about $5 \mathrm{~min}$. However, since the alignment 
and reconstruction are performed offline, after the tilt series has been acquired, it is difficult or even impossible to identify potential problems concerning the acquisition parameters or the sample conditions during the TEM experiment. Consequently, efficient optimization of the experimental settings of a 3D in situ experiment remains far from straightforward. Moreover, when studying nanoparticles by electron microscopy, it is of key importance to investigate those structures that are representative of the entire sample. On the other hand, sometimes very specific structures need to be selected for further characterization by TEM. Especially when the 3D structure of the nanoparticles is of importance, evaluating the relevance of a given particle for further investigation is difficult based on conventional, 2D TEM imaging. The ability to extract information about the 3D structure of a nanoparticle during its TEM investigation would enable the operator to immediately perform additional, optimized, or more detailed experiments on the same nanoparticle if necessary. One would be able to select, for example, particles with a specific 3D morphology prior to performing more advanced or detailed TEM experiments. Finally, to fully exploit the potential of in situ holders and novel acquisition methodologies, direct 3D visual feedback will be of essence to adjust the experimental parameters on-the-fly, in response to the observed dynamics of the nanoparticles. Clearly, realizing real-time 3D feedback would make a crucial impact in the field of 3D (in situ) characterization of nanoparticles, and may even become a standard for conventional TEM.

Here we report on a real-time workflow for electron tomography applied to nanoparticles, where alignment, reconstruction, and analysis are all carried out while acquiring the tilt series. The paper is structured as follows. In Section 2, we describe the concept of real-time reconstruction of arbitrary slices and how its implementation in the RECAST3D software has already enabled real-time synchrotron X-ray tomography. We also discuss which specific challenges have prevented using similar ideas for electron tomography. In Section 3, we propose the novel ingredients that we have used to extend the RECAST3D software for electron tomography. In Section 4, two case studies will be presented to illustrate that our approach enables a quantitative, real-time 3D characterization of the structure of complex nanoparticles. We also demonstrate the ability to quantitatively investigate their dynamic behavior in real-time during in situ experiments. Conclusions are presented in Section 5.

\section{Real-Time Reconstruction of Arbitrary Slices}

Recent developments concerning detector sensitivity and acquisition strategies have reduced the time to perform an entire acquisition for electron tomography from hours to several minutes or even less. As a consequence, there is an emerging need to develop reconstruction methodologies which can operate on the same time scale, that is, in real-time. Previous research has focused on accelerating electron tomography reconstructions by improving the reconstruction algorithms or enabling them to run in parallel on large computational clusters or on multiple GPUs. ${ }^{[2-31]}$ It has been reported that, when using a single NVIDIA GTX TITAN Z GPU, a $2048 \times 2048 \times 1024$ filtered back projection (FBP) reconstruction can be obtained within $\approx 10$ min. ${ }^{[32]}$ Such a short reconstruction time can be further reduced down to $2 \mathrm{~min}$ by making use of 4 GPUs. When more advanced iterative reconstruction algorithms such as the simultaneous iterative reconstruction technique (SIRT) ${ }^{[33]}$ are applied, capable of handling noisy and limited data, significantly longer computation times are required, even when using multiple GPUs. ${ }^{[32,34]}$

The computational load further increases when using stateof-the-art reconstruction algorithms, which exploit prior knowledge about the reconstructed object. The discrete algebraic reconstruction technique (DART), for instance, uses prior knowledge on the discrete density of an object. ${ }^{[35,36]}$ If the material consists out of homogeneous regions, separated by sharp interfaces, DART is able to produce accurate reconstructions from a limited number of projection images. Total variation minimization (TVM), on the other hand, includes knowledge on the sparsity of the discrete gradient of the reconstructed object to improve upon the reconstruction accuracy and facilitate further quantification. ${ }^{[37-40]}$ Although GPU implementations of reconstruction methods for 3D tomography do significantly accelerate the reconstruction process, the computational power of a single GPU is not enough to enable real-time 3D tomography, especially given the recent drive toward accelerated acquisition. Moreover, it has been demonstrated that, provided with dedicated post-processing, FBP-type of reconstruction algorithms can produce more accurate reconstructions in a significantly shorter time than iterative (regularized) methods. ${ }^{[41]}$ The recently to realize real-time reconstructions is to provide a dedicated GPU cluster for each electron microscope, which is complex and costly.

The recently developed reconstruction software RECAST3D provides an alternative way to realize real-time reconstructions. ${ }^{[32]}$ RECAST3D is available as open-source software ${ }^{[42]}$ under the GPL license and is based on the idea that inspecting a $3 \mathrm{D}$ reconstructed volume is typically carried out by slicing through the reconstructed volume in various suitable directions, effectively looking at a set of 2D slices. This approach requires a single workstation equipped with a powerful GPU, thereby avoiding the need for a complex and costly GPU-cluster setup. The software exploits the intrinsic speed of the FBP algorithm to reconstruct user-selected, arbitrarily oriented, 2D slices through the 3D structure in real time, without ever performing a full 3D volume reconstruction (Figure 1).

Although such slices are 2D images, they represent subsets of the 3D inner structure of the sample under investigation. A combination of different slices therefore yields quasi-3D information. Moreover, these slices can be computed at any arbitrary position and along any angle, enabling the TEM operator to dynamically highlight features of interest of the investigated object. Since reconstructing 2D slices is computationally far less expensive and significantly, more data efficient than reconstructing the entire 3D structure, slices can be automatically updated on the fly during the acquisition of a tilt series. While the experiment is ongoing, the user can dynamically translate and rotate the selected slices through the reconstruction, guided by a low resolution 3D preview of the full reconstruction, as demonstrated in Movie S1, Supporting Information. Consequently, the quasi-3D tomographic view can 


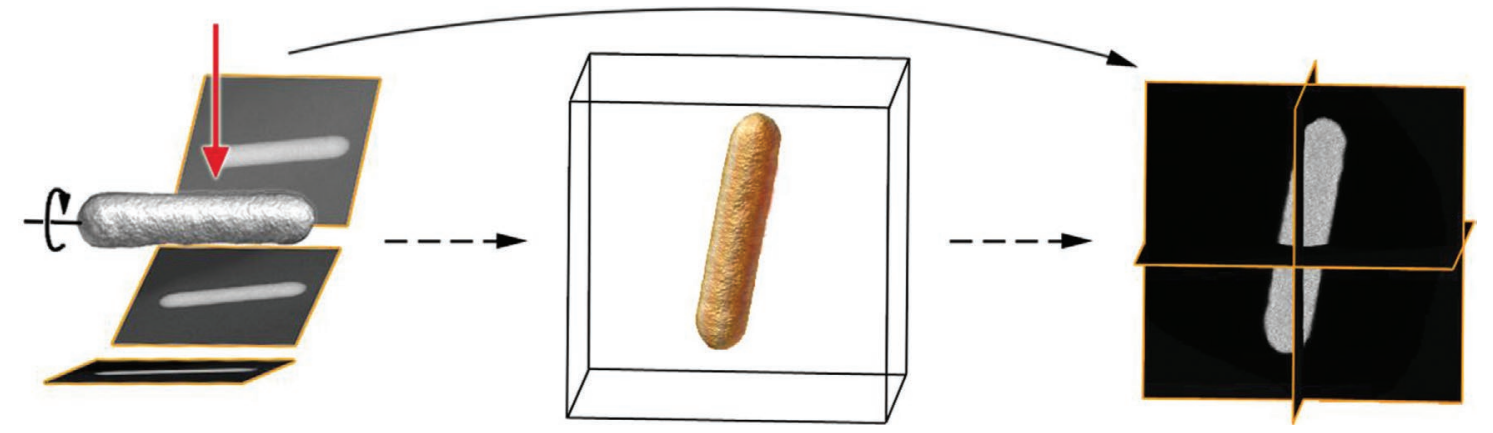

Figure 1. Illustration of the workflow of a conventional tomography experiment (dashed arrows). First, 2D projection images are acquired. The direction of the electron beam is indicated by the red arrow. Next, a complete 3D reconstruction is performed. Finally, orthogonal 2D slices through the 3D reconstruction are investigated. RECAST3D provides a new approach (solid line) in which user selected slices are reconstructed on demand.

be constructed in only a fraction of the time needed to acquire a regular $3 \mathrm{D}$ reconstruction of the entire structure, providing the groundwork for true real-time tomography.

The feasibility and usefulness of this approach has first been demonstrated for X-ray tomography at the TOMCAT beamline at the Swiss Light Source (PSI), where dynamic phenomena could be observed during imaging. ${ }^{[43]}$ Unfortunately, this concept cannot be applied to electron tomography in a straightforward manner because of several key differences between X-ray and electron tomography. Indeed, in X-ray tomography a large number of projection images are typically acquired over a complete angular range, resulting in an almost ideal sampling of the projection data space. Such data sets correspond exactly to the scenario where FBP provides accurate reconstructions. In contrast, in electron tomography, the risk of sample degradation or deformation during an electron tomography experiment makes it either impossible or undesirable to acquire a high amount of noise-free projection images. Therefore, the projection data are typically incomplete since the tilt increment is much larger (at least by a factor of 10) and the tilt range smaller (due to the missing wedge), as compared to X-ray tomography. In the case of imperfect projection data, because of either noise present in the projection images or when only a few projection images are available, FBP tends to result in imaging artefacts that hamper a quantitative interpretation of the reconstruction.

In the field of electron tomography, FBP is therefore typically outperformed by algebraic reconstruction methods such as the SIRT, ${ }^{[33]}$ which however are much slower than FBP and are not suitable for reconstructing arbitrarily oriented slices. ${ }^{[4-46]}$ Another key difference between electron tomography and X-ray tomography is the need for aligning the individual TEM projection images after their acquisition. Although several standard software packages are currently available for the alignment of electron tomography tilt series, none of them are able to perform the alignment in real-time. We introduce herein new computational ingredients that overcome all of these limitations and enable real-time reconstruction of arbitrary slices for electron tomography. These new components have been added to the open-source RECAST3D software, thereby making them available to the entire electron microscopy community. Next, we discuss the dedicated features that were incorporated into the RECAST3D reconstruction framework, specialized for electron tomography.

\section{Real-Time Reconstruction of Arbitrary Slices for Electron Tomography}

\subsection{Image Alignment}

When a new projection is acquired, it is aligned in real-time with respect to previous projections by first performing a center-ofmass correction based on the segmented projection image, using Otsu's thresholding method, ${ }^{[47]}$ followed by a conventional crosscorrelation method. This image alignment protocol is well-suited for aligning images of single nanoparticles. The center-of-mass correction shifts the center-of-mass of each projection image to the middle of that image and serves to provide a good starting point for the refined cross-correlation alignment. The cross-correlation between the subsequent projection images is computed as the normalized product between the Fourier transform of one image and the complex conjugate of the Fourier transform of the other. By locating the maximum in the cross-correlation, the relative shift between the images can be determined.

\subsection{Tilt Axis Alignment}

Since FBP relies on forward- and backprojections, it is important that the computational tilt axis is aligned to the experimental one. ${ }^{[48]}$ A possible discrepancy between both depends on the position of the particle of interest on the TEM grid or a slight bending of the grid and/or holder. Such discrepancy causes smearing artefacts in the reconstruction, lowering the reconstruction quality and hampering further quantification. Therefore, support was added for on-the-fly adjustment of the tilt axis alignment parameters. These adjustments are applied retroactively to the full set of projections. In this manner, the operator can manually translate and rotate the computational tilt axis and directly observe its effect on the reconstructed slices in order to optimally match it to the experimental tilt axis. These adjustments are applied to the modeled data geometry and leave the projection data untouched.

\subsection{Reconstruction}

RECAST3D relies on the computational efficiency of the FBP algorithm, which provides reliable 3D reconstructions for 
single-axis tomography, given that a sufficient number of high signal-to-noise projection images are available. For this discussion, let us assume we acquire $P$ projection images consisting of $N \times N$ pixels. The FBP algorithm for reconstructing a 3D volume of size $N \times N \times N$ comprises two steps. First, the data are filtered in Fourier space, which requires $O\left(P \times N^{2} \times \log N\right)$ operations. Next, the filtered data are backprojected onto the $3 \mathrm{D}$ volume. This backprojection step requires $O\left(P \times N^{3}\right)$ operations, and therefore dominates the computational complexity of the FBP algorithm. The voxel intensity $f_{\mathrm{FBP}}$ at position $(x, y, z)$ can therefore be retrieved as

$f_{\mathrm{FBP}}(x, y, z)=\int_{0}^{\pi} g_{\text {filtered }}(\theta, x \cos \theta+y \sin \theta, z) \mathrm{d} \theta$

with $g_{\text {filtered }}$ being the data after a 1D filter has been applied. This implies that, after filtering the projection data, the voxel intensity at any position $(x, y, z)$ can be computed directly from the filtered projections, independently of the rest of the $3 \mathrm{D}$ volume. This local property of FBP enables one to efficiently reconstruct any subset of the $3 \mathrm{D}$ volume directly from the filtered projection data.

In particular, backprojecting onto an arbitrarily oriented 2D slice consisting of $N \times N$ voxels can be performed in only $O\left(P \times N^{2}\right)$ operations. Since $N$ is typically in the range of $10^{3}$, this is significantly more efficient than a full 3D backprojection. The backprojections onto slices are therefore performed in the RECAST3D implementation as follows. Instead of backprojecting onto the whole $3 \mathrm{D}$ volume, a slab of size $N \times N \times 1$ is defined which coincides with the central axial slice of the acquisition geometry. To reconstruct a newly requested arbitrarily oriented slice, we modify the vectors associated to the acquisition geometry (the direction of the rays, as well as the detector position, and orientation) so that the requested slice is the central slice of the modified geometry. We then run a standard backprojection algorithm with the modified geometry and obtain the reconstruction for the requested slice. Modifying the geometry can be done efficiently, without ever changing the pre-filtered projection data. Using this method, any slice can be reconstructed from the filtered data with minimal overhead compared to a reconstruction of the central slice. Moreover, the filtering step can be performed in real time while acquiring projection images, and does not impact the reconstruction time for the chosen set of 2D slices, or when a new set of slices is selected for reconstruction.

Although we focus on single-axis tomography in this work, it is worth mentioning that the method discussed here can be applied for any acquisition geometry for which a method is available that has the same computational structure as FBP: a relatively inexpensive filtering step followed by a backprojection step. Such an FBP-like algorithm exists as well for instance for dual-axis tomography ${ }^{[49]}$ and laminography. ${ }^{[50]}$

Reconstruction results of a range of linear algebraic reconstruction methods can be accurately approximated by computing a so-called algebraic filter and using this computed filter in the FBP algorithm. ${ }^{[51]}$ The filter calculation itself is computationally intensive, but once determined, the resulting filter can be used with the same computational efficiency as FBP. To overcome the limitations of the classical FBP algorithm for electron tomography, support for algebraic filter methods was added to RECAST3D. By computing algebraic filters based on the SIRT algorithm and using these filters in FBP, arbitrary slices can now be reconstructed that are in close agreement with the output of SIRT. Other than the precomputation of the filters, which can be performed prior to the experiment, the reconstruction time is identical to standard FBP when this method is used. For the remainder of this work we will refer to this type of reconstruction as SIRT-FBP.

Adding these new components to the RECAST3D software enables real-time electron tomography, which will drastically optimize the efficiency of 3D characterization of nanomaterials, but more importantly will enable a new range of experiments such as real-time in situ electron tomography. As a proof of concept, we describe in what follows two different examples of electron tomography experiments wherein the benefits of this novel technique will be highlighted.

\section{Results and Discussion}

To achieve real-time visualization during electron tomography experiments, a workstation was coupled to the electron microscope, to function as a reconstruction server. This server received the tomographic projection images while being acquired at the electron microscope, via a 1 Gbps network connection and comprised an Intel Core i9-9900K CPU, 32 GB RAM, and a NVIDIA GeForce GTX 1070 GPU with 8 GB global memory. All results presented in this work were calculated on this workstation. The reconstruction pipeline comprises two asynchronous operations: a preprocessing operation and a reconstruction operation, both performed multiple times during the experiment. The first operation refers to the preprocessing of the projection images. This operation is performed immediately after a projection image is acquired, and is done completely independently from the reconstruction operation. The computationally most demanding part of this operation is to apply the algebraic filter, and to align the projection images. Using our implementation, the total time to perform this preprocessing operation is orders of magnitude shorter than the time required for the operator to acquire a projection image. Therefore, this operation is not a bottleneck for our real-time methodology. The second, reconstruction, operation is performed each time a new slice is chosen for visualization. Using the workstation described above, and for a dataset consisting of 51 projection images of $1024 \times 1024$ pixels, the total time elapsed from the moment a user selects a new slice, until the reconstruction shows in RECAST3D is $\approx 60 \mathrm{~ms}$.

\subsection{Explorative Quasi-3D Imaging}

As a first case study we investigate Au@Ag nanorods. Although $\mathrm{Au}$ nanorods have been widely studied as excellent anisotropic plasmonic nanomaterials, $\mathrm{Ag}$ is known to be a more efficient plasmonic metal. However, the lower chemical stability of $\mathrm{Ag}$ compared to $\mathrm{Au}$, leads to more complicated and less controlled nanoparticle synthesis methods. Therefore, efforts have been focused toward using pre-formed $\mathrm{Au}$ nanoparticles, 
such as penta-twinned bipyramids, as templates for the seeded growth of $\mathrm{Ag}$ nanorods. However, the specific 3D shape, chemical composition, and distribution of the different elements strongly affect the properties of the resulting core-shell nanoparticles. ${ }^{[13,52-54]}$ In particular, the plasmon resonances of core-shell nanoparticles depend sensitively on both edge and corner truncations present in the 3D structure, as well as on the core-shell geometry. Moreover, potential alloying between the constituent metals would also strongly influence the plasmonic response. ${ }^{[55,56]} 3 \mathrm{D}$ investigations of the structure and composition are therefore of critical importance to understand the properties of these nanoparticles.

To investigate the 3D distribution of $\mathrm{Au}$ and $\mathrm{Ag}$ in the core-shell nanoparticle, HAADF-STEM tomography is applied. The first step in such an electron tomography experiment is the selection of a representative nanoparticle with a suitable position and orientation on the grid. Conventionally, one can only decide if the investigated nanoparticle and its location are indeed suitable, once the $3 \mathrm{D}$ reconstruction has been completed offline. This, obviously, strongly reduces the efficiency of the experiment and leads to suboptimal use of microscopy time. Furthermore, the lack of real-time 3D feedback prevents the dynamic tuning of acquisition parameters during data collection, for example, to obtain a sufficient signal-to-noise ratio and/or resolution in the final reconstruction. Providing a fast quasi-3D image of the structure under investigation overcomes these limitations and enables a much more effective use of the TEM.

A HAADF-STEM projection image of one $\mathrm{Au@Ag}$ nanoparticle is presented in Figure 2a. A tilt series was acquired over an angular range of $\pm 72^{\circ}$ with a $9^{\circ}$ interval, using a Thermo Fisher Scientific Osiris electron microscope operated at $200 \mathrm{kV}$. Regions with different intensities can be clearly observed and an apparent rod-like shape of the nanoparticle is readily recognized. While the acquisition of the tilt series is ongoing, the projection images are instantly aligned and prefiltered. Simultaneously, arbitrarily oriented slices are reconstructed using the SIRT-FBP algorithm for which the filter was pre-computed to resemble 100 iterations of SIRT. Figure $2 \mathrm{~b}$ highlights the selected slices within the RECAST3D software.

Shown in Figure 2c is an enlarged quasi-3D visualization of the investigated structure, obtained after acquiring 17 projection images only. The background of the arbitrary oriented slices was set to transparent to enhance the $3 \mathrm{D}$ interpretation. The process described above, along with the selection of the slices is demonstrated in Movie S1, Supporting Information. Based on the real-time quasi-3D reconstruction one can immediately decide whether the selected particle is representative, for example, in this case whether the shape corresponds to a rod with pentagonal symmetry, and whether the missing wedge effect is within reasonable limits. Whereas it is impossible to determine the precise structure of the rod from a single projection image (Figure 2a), sharp facets, indicated by the green arrows, can be identified in the quasi-3D reconstruction. Furthermore, one can alter the acquisition parameters such as tilt interval, magnification, image size, beam current, to name a few, and immediately observe their influence on the reconstruction quality.

For example, in Figure $2 \mathrm{~d}$ an identical quasi-3D view is shown after decreasing the tilt interval from $9^{\circ}$ to $3^{\circ}$. Clearly this improves the contrast of the reconstruction, enabling a better qualitative interpretation of both the morphology and composition of the bimetallic nanoparticle. In this manner, one can determine from a limited number of projection images whether the particle under investigation is of interest, and if so decide how to optimize the acquisition parameters.

In addition to qualitative information, real-time quantitative information can be obtained as well. The RECAST3D software 

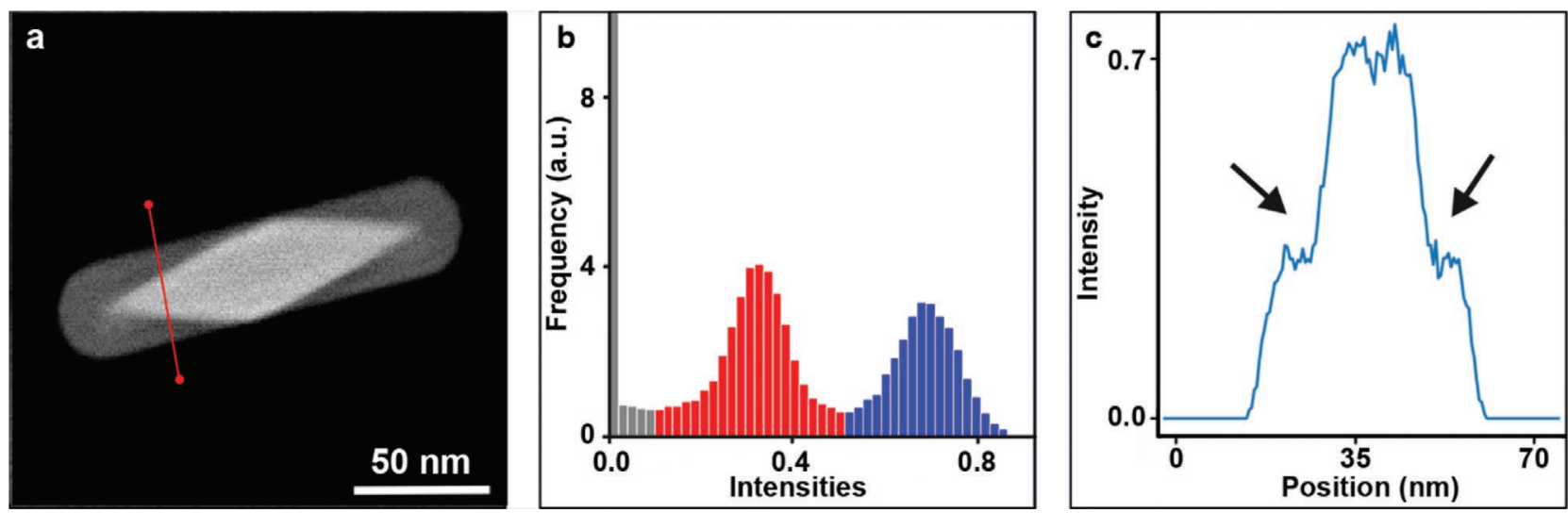

Figure 3. a) A central slice, reconstructed by SIRT-FBP for which the filter was pre-computed to resemble 100 iterations of SIRT. The profile is calculated along the line shown in red. b) Histogram of the central slice indicating the intensities corresponding to the background (gray), Ag (red), and Au (blue). c) Line profile through the slice. The sharp transition between $\mathrm{Au}$ and $\mathrm{Ag}$ is indicated by the black arrows.

has support for user-written Python plugins, which can be used to perform real-time analysis of specific features in the reconstructed 2D slices. For our example, we designed a plugin to evaluate the histogram and a line profile from a selected slice. Figure 3a illustrates the selected slice obtained from the SIRTFBP reconstruction. The histogram of the selected slice and a line profile through the slice are respectively shown in Figures 3b,c. The histogram bins (Figure 3b) corresponding to the background, Ag and Au signals, are respectively displayed in gray, red, and blue. The line across which the line profile is calculated, is indicated in red in Figure 3a.

It is possible to gain quantitative insight on the mixture of the $\mathrm{Au}$ and $\mathrm{Ag}$ phase by assessing the histogram and a line profile. Indeed, the histogram of the reconstructed intensities in Figure $3 \mathrm{~b}$ shows a clear segregation between the $\mathrm{Au}$ and $\mathrm{Ag}$ phase. This is confirmed by the sharp edges, indicated by black arrows, in the recorded line profile (Figure 3c). In addition, such line profiles reveal the shell thickness. Given that the distribution of the constituent metals and the size ratio between the core and shell strongly influence the plasmon response of the nanoparticles, the acquired knowledge is of great importance. Hence, on-the-fly access to arbitrary reconstructed slices opens the way to performing online quantitative data analysis. Moreover, in this case study, it would also enable the operator to select, for example, particles with a specific shell thickness for further TEM investigation of the plasmonic properties.

\subsection{Real-Time In Situ Tomography}

In addition to the advantages discussed above, the ability to visualize and analyze arbitrary slices in real time is ideal toward performing in situ 3D characterization by TEM. As an example of such an experiment, we present a 3D study of anisotropic $\mathrm{Au}$ nanostars. Because of their anisotropic shape and strong field enhancements at their tips, these nanostars are ideal substrates for plasmonics and surface-enhanced Raman spectroscopy (SERS). ${ }^{[8,57-59]}$ A representative 2D HAADF-STEM image of a nanostar is shown in Figure 4a. A well-known problem of such anisotropic nanostructures is their tendency to reshape at moderately high temperature, including photothermal effects related to intense laser irradiation. ${ }^{[60-63]}$ It is thus important to understand the deformation under these conditions, and to ultimately optimize their stability, which requires an accurate investigation of changes of the $3 \mathrm{D}$ structure during heating.

In our recent work, the morphological evolution of $\mathrm{Au}$ nanostars at elevated temperature was investigated by combining electron tomography with in situ heating. ${ }^{[25]}$ Although these experiments provided important information toward understanding the reshaping process, they were carried out in a rather inefficient manner. The main reason for this is that 2D HAADF-STEM projection images of such anisotropic nanoparticles are not sufficient to determine whether a specific nanostructure has the desired 3D morphology (e.g., a given number of branches), prior to starting the 3D in situ experiment. More importantly, if the $3 \mathrm{D}$ reconstruction is performed offline, it is nearly impossible to monitor how the structure changes as a function of the external conditions (e.g., temperature, heating time, etc.) while the experiment is ongoing, especially for highly anisotropic nanoparticles. Since the RECAST3D methodology yields real-time and quasi-3D information on the investigated structure, these limitations can be overcome.

The experiments were performed using a DENSsolutions wildfire heating holder and a Thermo Fisher Scientific Osiris electron microscope operated at $200 \mathrm{kV}$. A tomography series was acquired at room temperature, over a tilt range of $\pm 75^{\circ}$ with a $3^{\circ}$ increment. Figure $4 \mathrm{~b}$ shows the orientation of the chosen slices. Each slice was reconstructed using the SIRT-FBP algorithm, for which the filter was pre-computed to resemble 100 iterations of SIRT. As mentioned above, the position and orientation of the slices can be adapted on-the-fly to investigate features of the nanoparticle that are of particular interest. By exploring the 3D structure of the nanoparticle in this manner, eight sharp branches were identified, seven of which can be seen in the single quasi-3D view presented in Figure 4c. The selection of the slices and the identification of the different branches is demonstrated in Movie S2, Supporting Information. Since it is expected that the most apparent morphological changes will occur at sharp branches, ${ }^{[64-66]}$ we propose that this particular nanostar is an ideal candidate for the in situ experiment. This is not always obvious from a single HAADF-STEM 

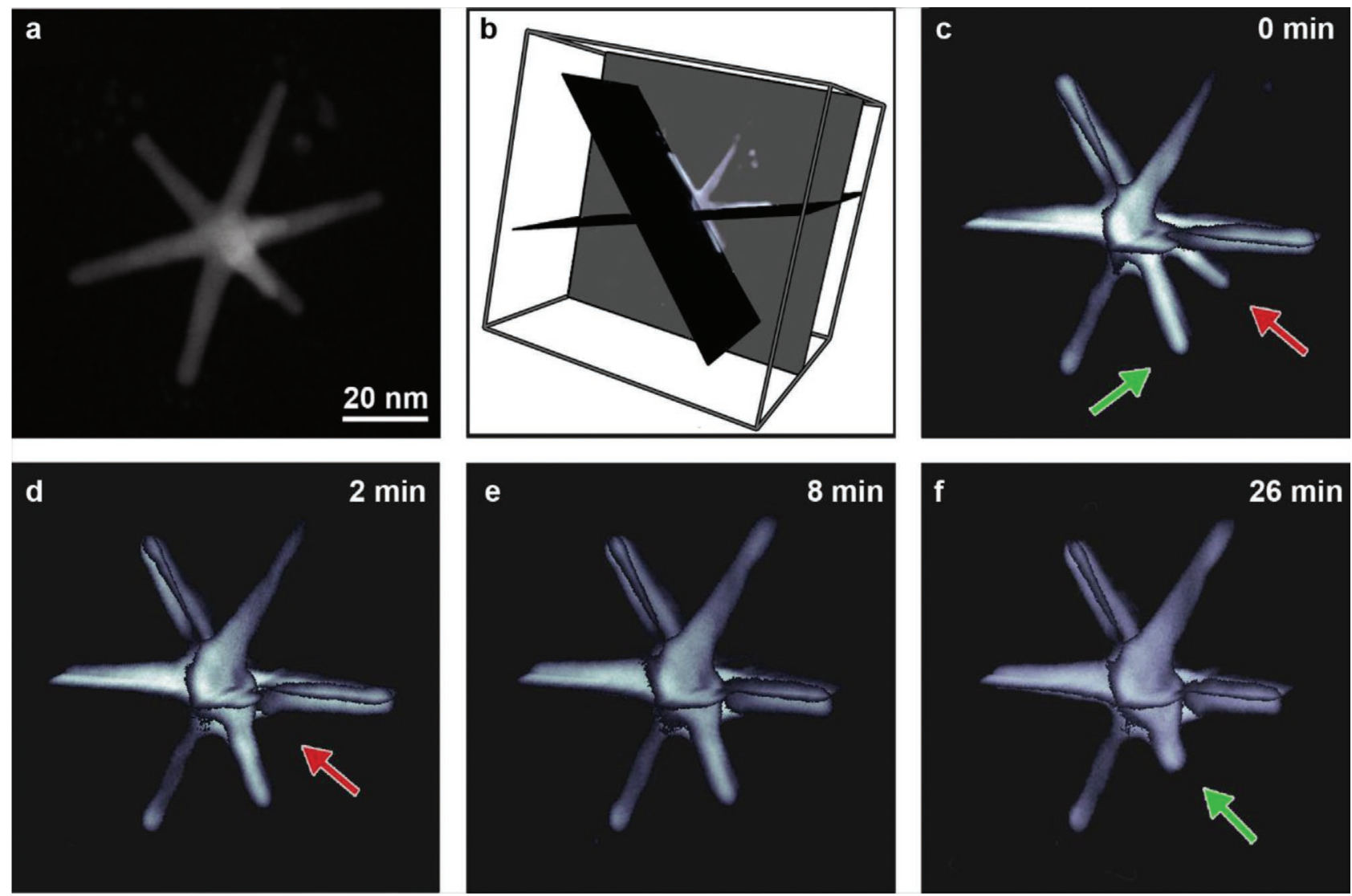

Figure 4. a) 2D HAADF-STEM image of a Au nanostar. b) 3D depiction of the selected slices reconstructed by SIRT-FBP, for which the filter was precomputed to resemble 100 iterations of SIRT. c-f) Quasi-3D view of the SIRT-FBP reconstruction of the nanostar after respectively 0, 2, 8, and 26 min of accumulated heating at $300^{\circ} \mathrm{C}$.

projection, since it is only a $2 \mathrm{D}$ projection of the true $3 \mathrm{D}$ structure, which can be very misleading.

To initiate the morphological transition, the nanostar was heated at $300{ }^{\circ} \mathrm{C}$ in different steps for the duration of 2, 6, and $18 \mathrm{~min}$. The first heating step was chosen to last for $2 \mathrm{~min}$ only, as it has been previously demonstrated that most heatinduced deformation occurs within the first minutes. ${ }^{[27,28,67]} \mathrm{We}$ decided on the duration of subsequent heating steps by using the immediate feedback provided by RECAST3D. After each heating step, the nanoparticle was quenched to room temperature to temporarily interrupt the morphological evolution and to acquire a HAADF-STEM tomographic series using the settings described above.

Visual inspection of the quasi-3D image in Figure 4d tells us that after only $2 \mathrm{~min}$ of heating, the nanostar has already deformed. We observe that several of the branches underwent a transformation, in agreement with earlier work, from a long sharp morphology to a broad and shorter shape with a more rounded tip. ${ }^{[27]}$ The lower right branch at the back of the nanostar, indicated by a red arrow, almost completely disappears after $2 \mathrm{~min}$ of heating. Direct contact of this tip with the support during the experiment might explain this behavior, as was observed as well in earlier studies. ${ }^{[28]}$ However, the fast feedback provided by the set of slices indicates that the change of all other branches remains relatively limited. Therefore, we decided to increase the duration of the heat treatment to $6 \mathrm{~min}$ during the next heating step. From Figure 4e, it can be seen that subsequent heating results in further shrinkage of the branches, during which the volume redistributes along their surface. We do observe that the deformation slows down, which can be attributed to the interaction between the electron beam and the ligands surrounding the nanostar, forming a protective carbon shell. ${ }^{68]}$ Based on these immediate results, we decided to heat the nanoparticle once more for $18 \mathrm{~min}$. From Figure 4f, it is clear that reshaping has stagnated and that except for the shrinkage of the foremost branch (indicated by the green arrow), only minor additional changes can be appreciated.

RECAST3D can also be used to extract quantitative information in real time. For the branches of nanostars, we anticipate that the curvature would decrease as a function of heating time. By monitoring curvature changes, we can quantify how much the nanostar reshapes over time and consequently evaluate the pace of the morphological evolution and whether it has been completed or not. Details on the curvature calculation are provided in the Supporting Information. In Figure 5a, histograms of the calculated positive curvature values are presented. The analysis was performed on a specifically chosen slice, away from contact with the heating chip.

From the histograms, which can be extracted in real time during the in situ experiment, it is possible to discern a shift from the high curvature values to lower values over the course of heating. Figure $5 \mathrm{~b}$ displays the maximal curvature as a 
a

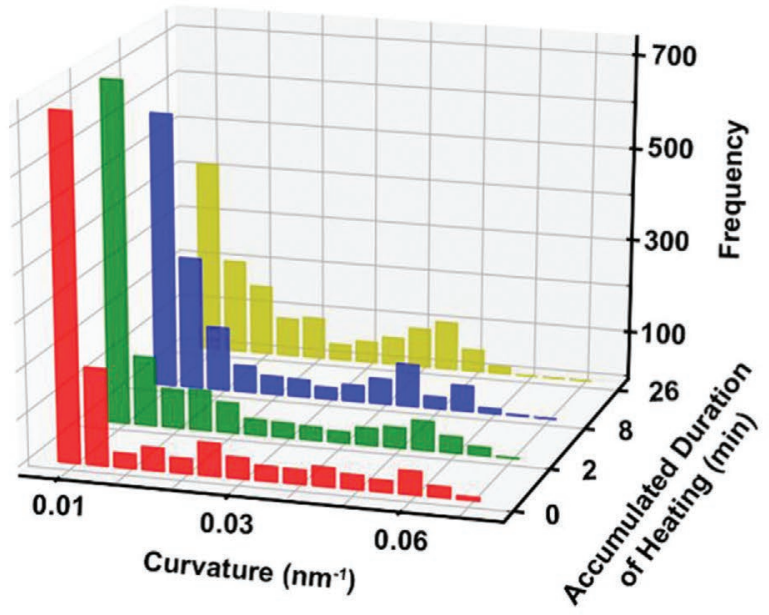

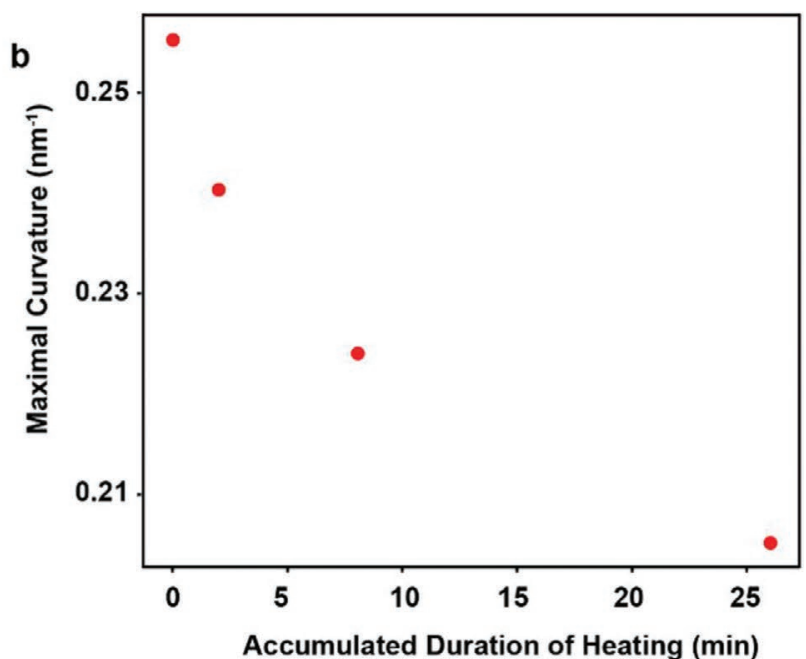

Figure 5. a) Histograms of the obtained curvature values from RECAST3D. b) The maximal curvature of one slice plotted as a function of the duration of heat treatment.

function of the accumulated duration of heating. It is apparent that the morphological evolution can be directly monitored from the change in maximal curvature. Most of the transition occurs at the first heating steps, during which the long thin branches lose their sharp tips and become blunt. After the first $8 \mathrm{~min}$ of heating, the decline in maximal curvature slows down, indicating that the amount of morphological evolution decreases. After $26 \mathrm{~min}$ of heating, the volume redistribution has almost stopped. Such an active feedback is crucial to adaptively control the parameters of an in situ investigation. This example shows that quasi-3D reconstructions can be used to provide real-time qualitative and quantitative information. In this manner, all experimental parameters can be efficiently tuned while the experiment is ongoing. Consequently, the operator is able to investigate a higher number of nanoparticles during a given microscopy session, leading to more statistically significant information.

\section{Conclusion}

We propose a new approach to compute high quality 2D slices through nanoparticles in real time, based on electron tomography tilt series. This technique is of great importance to improve the efficiency of $3 \mathrm{D}$ characterization of nanomaterials by TEM. It enables explorative imaging and provides valuable information to dynamically adjust the acquisition parameters during an electron tomography experiment. Moreover, quantification of specific features of nanoparticles becomes possible in real time, even while performing in situ experiments. We therefore consider the ability to gain real-time quasi-3D visualizations as the next (r)evolution in the field of 3D characterization of nanomaterials.

\section{Experimental Section}

Chemicals: Tetrachloroauric acid trihydrate $\left(\mathrm{HAuCl}_{4} \cdot 3 \mathrm{H}_{2} \mathrm{O}\right.$, $\geq 99 \%)$, citric acid $(\geq 99.5 \%)$, sodium borohydride $\left(\mathrm{NaBH}_{4}, 99 \%\right)$,
L-ascorbic acid ( $\geq 99 \%)$, silver nitrate $\left(\mathrm{AgNO}_{3}, \geq 99 \%\right)$, TritonX-100, hexadecyltrimethylammonium bromide (CTAB, $\geq 99 \%)$ and hexadecyltrimethylammonium chloride (CTAC, $25 \mathrm{wt} \%$ in water), were purchased from Sigma-Aldrich. Hydrochloric acid solution (37 wt\%) was purchased from Fisher Chemical. All chemicals were used without further purification. Milli-Q water (resistivity $18.2 \mathrm{M} \Omega \mathrm{cm}$ at $25^{\circ} \mathrm{C}$ ) was used in all experiments. All glassware were cleaned with aqua regia, rinsed with Milli-Q water, and dried before use.

Synthesis of Au@Ag Core-Shell Nanorods with Pentagonal Symmetry: The Au@Ag nanorod sample was prepared using Au nanobipyramiddirected $\mathrm{Ag}$ growth, modified from a previous report. ${ }^{[69,70]}$ For the synthesis of Au nanobipyramids, a freshly prepared $\mathrm{NaBH}_{4}$ solution $(0.025 \mathrm{M}, 0.25 \mathrm{~mL})$ was mixed with an aqueous solution composed of $\mathrm{HAuCl}_{4}(1 \mathrm{~mm}, 2.5 \mathrm{~mL})$, citric acid $(0.02 \mathrm{M}, 2.5 \mathrm{~mL})$, and CTAC $(0.1 \mathrm{M}$, $5 \mathrm{~mL}$ ) under vigorous stirring. This seed solution was aged in a water bath at $80^{\circ} \mathrm{C}$ for $90 \mathrm{~min}$, under stirring. The aged seed solution $(0.1 \mathrm{~mL})$ was injected into an aqueous growth solution containing CTAB $(0.1 \mathrm{M}$, $10 \mathrm{~mL}), \mathrm{HAuCl}_{4}(0.01 \mathrm{M}, 0.5 \mathrm{~mL}), \mathrm{AgNO}_{3}(0.01 \mathrm{M}, 0.1 \mathrm{~mL}), \mathrm{HCl}(1 \mathrm{M}$, $0.2 \mathrm{~mL})$, and ascorbic acid $(0.1 \mathrm{M}, 0.08 \mathrm{~mL})$, under vigorous stirring. The reaction solution was kept in a water bath at $30^{\circ} \mathrm{C}$ for $4 \mathrm{~h}$ under stirring. For $\mathrm{Ag}$ overgrowth, the Au nanobipyramid solution was centrifuged at $4200 \mathrm{rpm}(1950 \mathrm{~g})$ for $20 \mathrm{~min}$ and redispersed in CTAC $(0.01 \mathrm{M}, 5 \mathrm{~mL})$, followed by subsequent addition and mixing of $\mathrm{AgNO}_{3}(0.01 \mathrm{M}, 1.4 \mathrm{~mL})$ and ascorbic acid $(0.1 \mathrm{M}, 0.7 \mathrm{~mL})$.

The solution was kept in a water bath at $60^{\circ} \mathrm{C}$ for $2 \mathrm{~h}$ under stirring, during which $\mathrm{Ag}$ was overgrown on the Au nanobipyramids to form nanorod-shaped nanoparticles. The as-prepared Au@Ag nanorods were centrifuged at $3000 \mathrm{rpm}(990 \mathrm{~g})$ for $20 \mathrm{~min}$ and redispersed in water.

Synthesis of $\mathrm{Au}$ Nanostars: Au nanostars were prepared using a modified seed mediated method. ${ }^{[7]}$ Briefly, a seed solution was prepared by adding freshly prepared $\mathrm{NaBH}_{4}(0.6 \mathrm{~mL} ; 10 \mathrm{~mm})$ to a mixture of $\mathrm{HAuCl}_{4}(0.05 \mathrm{~mL} ; 50 \mathrm{~mm})$, and TritonX-100 (10 mL; $\left.0.15 \mathrm{M}\right)$, under stirring. The color of the seed solution changed from pale yellow to orange after addition of $\mathrm{NaBH}_{4}$. This seed solution was continuously stirred for $2 \mathrm{~min}$ and then aged for $10 \mathrm{~min}$ at room temperature. $24 \mu \mathrm{L}$ of the above-mentioned seed solution was then added under vigorous stirring to the growth solution consisting of $\mathrm{HAuCl}_{4}(0.2 \mathrm{~mL} ; 50 \mathrm{~mm})$, TritonX-100 (20 mL;0.15 M), ascorbic acid (0.08 mL; $0.8 \mathrm{M})$, and $\mathrm{AgNO}_{3}$ $(0.16 \mathrm{~mL} ; 10 \mathrm{~mm})$. The growth solution was then stirred for an hour. Further, to stabilize the nanostar morphology, $0.3 \mathrm{~mL}$ of freshly prepared $0.1 \mathrm{~mm}$ PEG-SH $\left(M_{\mathrm{w}} 5000\right)$ was added to the growth solution, followed by an hour of additional stirring. As-synthesized Au nanostars were centrifuged twice at $5000 \mathrm{rpm}$ for $15 \mathrm{~min}$ and redispersed in $2 \mathrm{~mL}$ of Milli-Q water. 


\section{Supporting Information}

Supporting Information is available from the Wiley Online Library or from the author.

\section{Acknowledgements}

H.V. and J.-W.B. contributed equally to this work. H.V. acknowledges financial support by the Research Foundation Flanders (FWO grant $1532617 N$ ). S.B. acknowledges financial support by the Research Foundation Flanders (FWO grant G026718N). Financial support was provided by The Netherlands Organization for Scientific Research (NWO), project numbers 639.073.506 and 016.Veni.192.235. This project received funding as well from the European Union's Horizon 2020 research and innovation program under grant agreement No. 731019 (EUSMI) and No. 815128 (REALNANO).

\section{Conflict of Interest}

The authors declare no conflict of interest.

\section{Keywords}

HAADF-STEM, in situ heating, nanoparticles, plasmonics, real-time tomography

Received: March 06, 2020

Revised: May 04, 2020

Published online: May 29, 2020

[1] W. L. Barnes, A. Dereux, T. W. Ebbesen, Nature 2003, 424, 824.

[2] K. L. Kelly, E. Coronado, L. L. Zhao, G. C. Schatz, J. Phys. Chem. B 2003, 107, 668.

[3] A. S. Aricò, P. Bruce, B. Scrosati, J. M. Tarascon, W. Van Schalkwijk, Nat. Mater. 2005, 4, 366.

[4] M. Pelton, J. Aizpurua, G. Bryant, Laser Photonics Rev. 2008, 2, 136.

[5] P. S. Kumar, I. Pastoriza-Santos, B. Rodríguez-González, F. J. G. De Abajo, L. M. Liz-Marzán, Nanotechnology 2008, 19, 015606.

[6] R. Sardar, A. M. Funston, P. Mulvaney, R. W. Murray, Langmuir 2009, 25, 13840.

[7] S. Linic, P. Christopher, D. B. Ingram, Nat. Mater. 2011, 10, 911.

[8] A. Guerrero-Martínez, S. Barosa, I. Pastoriza-Santos, L. M. Liz-Marzán, Curr. Opin. Colloid Interface Sci. 2011, 16, 118.

[9] S. Linic, U. Aslam, C. Boerigter, M. Morabito, Nat. Mater. 2015, 14, 567.

[10] A. Espinosa, A. K. A. Silva, A. Sánchez-Iglesias, M. Grzelczak, C. Péchoux, K. Desboeufs, L. M. Liz-Marzán, C. Wilhelm, Adv. Healthcare Mater. 2016, 5, 1040.

[11] S. J. Oldenburg, R. D. Averitt, S. L. Westcott, N. J. Halas, Chem. Phys. Lett. 1998, 288, 243.

[12] C. J. Desantis, R. G. Weiner, A. Radmilovic, M. M. Bower, S. E. Skrabalak, J. Phys. Chem. Lett. 2013, 4, 3072.

[13] W. Albrecht, J. E. S. Van Der Hoeven, T. S. Deng, P. E. De Jongh, A. Van Blaaderen, Nanoscale 2017, 9, 2845.

[14] J. Radon, Ber. Sachs. Akad. der Wiss. Leipzig J. Math. Phys. 1917, 69, 71.

[15] R. G. Hart, Science 1968, 159, 1464.

[16] W. Hoppe, R. Langer, G. Knesch, C. Poppe, Die Naturwiss. 1968, 55, 333.
[17] R. Gordon, R. Bender, G. T. Herman, J. Theor. Biol. 1970, 29, 471.

[18] P. A. Midgley, M. Weyland, Ultramicroscopy 2003, 96, 413.

[19] P. A. Midgley, R. E. Dunin-Borkowski, Nat. Mater. 2009, 8, 271.

[20] C. Kübel, A. Voigt, R. Schoenmakers, M. Otten, D. Su, T. C. Lee, A. Carlsson, J. Bradley, Microsc. Microanal. 2005, 11, 378.

[21] P. A. Midgley, E. P. W. Ward, A. B. Hungría, J. M. Thomas, Chem. Soc. Rev. 2007, 36, 1477.

[22] G. Möbus, B. J. Inkson, Mater. Today 2007, 10, 18.

[23] G. Van Tendeloo, S. Bals, S. Van Aert, J. Verbeeck, D. Van Dyck, Adv. Mater. 2012, 24, 5655.

[24] S. Bals, B. Goris, L. M. Liz-Marzán, G. Van Tendeloo, Angew. Chem., Int. Ed. 2014, 53, 10600.

[25] V. Migunov, H. Ryll, X. Zhuge, M. Simson, L. Strüder, K. J. Batenburg, L. Houben, R. E. Dunin-Borkowski, Sci. Rep. 2015, 5, 14516.

[26] L. Roiban, S. Li, M. Aouine, A. Tuel, D. Farrusseng, T. Epicier, J. Microsc. 2018, 269, 117.

[27] H. Vanrompay, E. Bladt, W. Albrecht, A. Béché, M. Zakhozheva, A. Sánchez-Iglesias, L. M. Liz-Marzán, S. Bals, Nanoscale 2018, 10, 22792.

[28] W. Albrecht, E. Bladt, H. Vanrompay, J. D. Smith, S. E. Skrabalak, S. Bals, ACS Nano 2019, 13, 6522.

[29] W. J. Palenstijn, K. J. Batenburg, J. Sijbers, J. Struct. Biol. 2011, 176, 250.

[30] W. Xu, F. Xu, M. Jones, B. Keszthelyi, J. Sedat, D. Agard, K. Mueller, J. Struct. Biol. 2010, 171, 142.

[31] V. V. Nikitin, F. Andersson, M. Carlsson, A. A. Duchkov, Comput. Geosci. 2017, 105, 21.

[32] J. W. Buurlage, H. Kohr, W. Jan Palenstijn, K. Joost Batenburg, Meas. Sci. Technol. 2018, 29, 064005.

[33] P. Gilbert, J. Theor. Biol. 1972, 36, 105.

[34] J. W. Buurlage, R. H. Bisseling, K. J. Batenburg, Parallel Comput. 2019, 81, 104.

[35] K. J. Batenburg, S. Bals, J. Sijbers, C. Kübel, P. A. Midgley, J. C. Hernandez, U. Kaiser, E. R. Encina, E. A. Coronado, G. Van Tendeloo, Ultramicroscopy 2009, 109, 730.

[36] K. J. Batenburg, J. Sijbers, IEEE Trans. Image Process. 2011, 20, 2542.

[37] B. Goris, W. Van den Broek, K. J. Batenburg, H. Heidari Mezerji, S. Bals, Ultramicroscopy 2012, 113, 120.

[38] R. Leary, Z. Saghi, P. A. Midgley, D. J. Holland, Ultramicroscopy 2013, $131,70$.

[39] R. Leary, Z. Saghi, P. A. Midgley, D. J. Holland, Microsc. Microanal. 2013, 19, 546.

[40] B. Goris, T. Roelandts, K. J. Batenburg, H. H. Mezerji, S. Bals, Ultramicroscopy 2013, 20, 766.

[41] D. M. Pelt, K. J. Batenburg, J. A. Sethian, J. Imaging 2018, 4, 128.

[42] J.-W. Buurlage, W. J. Palenstijn, A. Hendriksen, D. Pelt, A. Graas, H. Kohr, Zenodo 2020, https://doi.org/10.5281/ZENODO.3773540.

[43] J. W. Buurlage, F. Marone, D. M. Pelt, W. J. Palenstijn, M. Stampanoni, K. J. Batenburg, C. M. Schlepütz, Sci. Rep. 2019, 9, 18379.

[44] F. Pontana, A. Duhamel, J. Pagniez, T. Flohr, J. B. Faivre, A. L. Hachulla, J. Remy, M. Remy-Jardin, Eur. Radiol. 2011, 21, 636.

[45] F. Pontana, J. Pagniez, T. Flohr, J. B. Faivre, A. Duhamel, J. Remy, M. Remy-Jardin, Eur. Radiol. 2011, 21, 627.

[46] D. Chen, B. Goris, F. Bleichrodt, H. H. Mezerji, S. Bals, K. J. Batenburg, G. de With, H. Friedrich, Ultramicroscopy 2014, 147, 137.

[47] N. Otsu, IEEE Trans. Syst., Man, Cybern. 1979, 9, 62.

[48] M. Hayashida, S. Ogawa, M. Malac, Microsc. Res. Tech. 2018, 81, 515.

[49] D. N. Mastronarde, J. Struct. Biol. 1997, 120, 343. 
[50] A. Myagotin, A. Voropaev, L. Helfen, D. Hänschke, T. Baumbach, IEEE Trans. Image Process. 2013, 22, 5348.

[51] D. M. Pelt, K. J. Batenburg, Int. Meet. Fully Three-Dimensional Image Reconstr. Radiol. Nucl. Med. 2015, 158.

[52] S. H. Joo, J. Y. Park, C. K. Tsung, Y. Yamada, P. Yang, G. A. Somorjai, Nat. Mater. 2009, 8, 126.

[53] M. Shao, Q. Chang, J. P. Dodelet, R. Chenitz, Chem. Rev. 2016, 116, 3594.

[54] G. Guisbiers, R. Mendoza-Cruz, L. Bazán-Díaz, J. J. Velázquez-Salazar, R. Mendoza-Perez, J. A. Robledo-Torres, J. L. Rodriguez-Lopez, J. M. Montejano-Carrizales, R. L. Whetten, M. José-Yacamán, ACS Nano 2016, 10, 188.

[55] M. B. Cortie, A. M. McDonagh, Chem. Rev. 2011, 111, 3713.

[56] H. Jing, Q. Zhang, N. Large, C. Yu, D. A. Blom, P. Nordlander, H. Wang, Nano Lett. 2014, 14, 3674.

[57] F. Hao, C. L. Nehl, J. H. Hafner, P. Nordlander, Nano Lett. 2007, 7 , 729.

[58] H. Yuan, C. Khoury, A. Fales, C. Wilson, G. Grant, T. Vo-Dinh, Biomed. Opt. 2012, 2012, BM2A.8.

[59] X. T. Kong, Z. Wang, A. O. Govorov, Adv. Opt. Mater. 2017, 5, 1600594.

[60] G. Baffou, R. Quidant, Laser Photonics Rev. 2013, 7, 171.
[61] G. Baffou, R. Quidant, C. Girard, Appl. Phys. Lett. 2009, 94, 153109.

[62] L. Cao, D. N. Barsic, A. R. Guichard, M. L. Brongersma, Nano Lett. 2007, 7, 3523.

[63] G. Baffou, H. Rigneault, Phys. Rev. B 2011, 84, 035415.

[64] A. B. Taylor, A. M. Siddiquee, J. W. M. Chon, ACS Nano 2014, 8, 12071.

[65] S. Inasawa, M. Sugiyama, Y. Yamaguchi, J. Phys. Chem. B 2005, 109, 3104.

[66] S. Link, C. Burda, B. Nikoobakht, M. A. El-Sayed, J. Phys. Chem. B 2000, 104, 6152

[67] H. Petrova, J. P. Juste, I. Pastoriza-Santos, G. V. Hartland, L. M. Liz-Marzán, P. Mulvaney, Phys. Chem. Chem. Phys. 2006, 8, 814.

[68] W. Albrecht, A. van de Glind, H. Yoshida, Y. Isozaki, A. Imhof, A. van Blaaderen, P. E. de Jongh, K. P. de Jong, J. Zečević, S. Takeda, Ultramicroscopy 2018, 193, 97.

[69] A. Sánchez-Iglesias, N. Winckelmans, T. Altantzis, S. Bals, M. Grzelczak, L. M. Liz-Marzán, J. Am. Chem. Soc. 2017, 139, 107.

[70] Z. J. Yang, R. Jiang, X. Zhuo, Y. M. Xie, J. Wang, H. Q. Lin, Phys. Rep. 2017, 701, 1.

[71] S. Atta, M. Beetz, L. Fabris, Nanoscale 2019, 11, 2946. 